\title{
TIME ESTIMATION IMPROVEMENT IN AGILE DEVELOPMENT
}

\author{
Willem van der Feltz ${ }^{1}$, Niels van der Pas ${ }^{2}$ and Mark van der Pas ${ }^{3}$ \\ ${ }^{1}$ European Center for Machine Learning, Gutenbergstraße 21, 50823 Köln, Germany \\ ${ }^{2}$ European Center for Machine Learning, Willem II singel 2, 6041HS, Roermond, The Netherlands \\ ${ }^{3}$ European Center for Digital Transformation, Willem II singel 2, 6041HS, Roermond, The Netherlands
}

\begin{abstract}
Estimating the time needed to execute an IT project has turned out to be a major challenge in daily praxis. Despite high inaccuracies, these estimations are important as they can be used to determine the cost for offering an IT project and in deciding whether to execute it at all. In this study we focused on the estimations for user stories created by a European Software company delivering $100 \%$ of their software in an agile way of working. Over a time-span of 10 months, 1738 estimations were created on small user stories (around 5 hours of work on average). Three main conclusions could be drawn from the data. Firstly, it was found that smaller-than-two-hour user stories were over-estimated roughly as frequently as under-estimated. In contrast, estimations for larger-than-two-hour user stories were less balanced and on average too low (under-estimated). Secondly, and surprisingly, we did not see that estimations were better when the estimator was able to execute the task himself versus when he was not. The statistics suggest that estimators who are able to do the task themselves tend to underestimate the time needed to deliver the user story. Finally, we found that the accuracy of estimations does not improve over time.
\end{abstract}

\section{KEYWORDS}

IT Projects, IT Portfolio Management, Plan Accuracy, Deadlines, Decision Support

\section{INTRODUCTION}

Delivering projects on time remains a major challenge. Nowadays, a central part of many big projects is software development. Delays and complications in this field can have big effects on the overall project. Recent examples of time overrun due to software problems come from the London Crossrail delaying the schedule from 2018 to 2021 (Farrel \& Topham, 2019) or from the Smart Energy meter pushing its deadline back more than four years into 2024 (Ambrose, 2019). A third example comes from a newly identified software flaw in the grounded Boeing 737 Max (Levin \& Schlangenstein, 2020). More recent examples can be found in a new paper by S. Lauesen (Lauesen, 2020).

This challenge, delivering IT projects on time, is not new and it has been monitored for more than two decades by the Standish Group. In their 2015 research, they found that $60 \%$ of IT projects are not finished on time (The Standish Group, 2015).

Finishing projects requires the successful execution of hundreds, thousands and sometimes millions of small activities. The time needed for each of these steps can be estimated and those estimations can be compared to the actual time needed. The accuracy of these estimations is likely to go down in case of a large amount of interdependent activities planned with a bias and over multiple years. Developing software in an agile way (e.g. (Hoffmann, et al., 2020)) reduces the need to plan over long time spans as well as the size of the individual activities.

An important part in the flow of a project is making estimations of how long things will take and, very much related, how much they will cost. Part of the above problem could be solved by becoming better at making estimations. Estimation accuracy has been the subject of a lot of research in the past (Boehm, 1984), and still is today (Bilal, et al., 2020). 
In this research we focus on time estimations made for smaller IT programming tasks executed within an agile work setting, taking around 5 hours on average. We will use the words user story to describe these tasks. We consider two aspects: 1 . estimating the time a user story will take and 2 . how well the user story is delivered within the estimated/allocated time. We will study the accuracy of time estimations for different categories (or reference classes) of user stories as well as the usage of feedback instruments to improve this accuracy.

This paper discusses the theoretical foundations and associated hypotheses. Then the research method is outlined and results are presented. Finally, the conclusions, limitations and suggestions for future research are presented.

\section{THEORY AND HYPOTHESES}

Goldratt (Goldratt, 2004) proposes several actions to improve on time (and in budget) deliveries. Amongst others he proposes to:

1. split work into small tasks

2. assure accurate estimations created by experts

3. openly discuss the time needed for a task

4. plan buffer at the end of the critical chain

5. report daily progress

6. protect the critical chain

In this study we focus on the first two topics. User stories can differ significantly in size but can, as compared to complete IT projects, be seen as 'small tasks'. Goldratt suggests splitting a project into small tasks to force the team to list all steps needed. This avoids some tasks being overlooked, helping with the overall estimation accuracy. Splitting up could also help because the estimation accuracy for small user stories could be higher than that of larger user stories: small user stories are easier to oversee. To use a metaphor for this argument: estimating the time needed to travel to the nearest city is probably more accurate than estimating the time needed to travel to Timbuktu, assuming that is not your nearest city. This brings us to our first hypothesis

H1: Plan accuracy of time estimations for smaller-than-two-hour user stories are more accurate than those for larger user stories.

Goldratt suggests that the estimations should be created by an experienced person, preferably a colleague who will execute the task. Expert-based estimation of software development has been investigated quite extensively and is often considered on equal footing with formal, model-based estimations (Mahmood, et al., 2020). To build on the earlier metaphor: in case you know how to travel to the nearest city, then you can more accurately estimate the time needed to do so. On the other hand the 'expert blind spot' (Wiggins \& McTighe, 2005) indicates that the expert will underestimate the time as it is complex to correct the estimation for the experience difference between the estimator and the person delivering the work. This blind spot is especially expected when there is a large difference in expertise. As this is more the case in educational settings and expectedly less in professional settings, we come to the second hypothesis.

H2: The plan accuracy of estimations is higher in case the estimator can execute the task himself.

Thirdly, perhaps most straightforwardly, we can look at the time-component of the data and test whether estimators improve accuracy over time. To build on the earlier metaphor: in case you have already estimated time to travel to several cities and received feedback on these estimations, then you can more accurately estimate the time needed to do so the next time.

For estimators to be able to improve, some form of feedback is needed. This can be done by giving estimators basic feedback on the actual time spend as compared to their estimation (Jørgensen, 2004). This feedback can be provided for each estimation separately. As estimators learn over time based on their previous estimations, we expect their accuracy to improve. Even though these improvements are not always present in IT, e.g. in a paper on the predictability of IT investment business value (van der Pas \& Furneaux, 2015) it was concluded that estimates of the value of projects do not improve over time, we nevertheless expect that:

H3: Plan Accuracy of estimations of an estimator improves over time.

In the next section we present the methods used to test these hypotheses. 


\section{METHOD}

Our research is based on data and experience from an international software organisation with customers in Europe and on-site operations in four European countries. A part of that organization shared estimations for the work they do in an agile Kanban organized structure. In total, 2086 user story estimations were offered for analysis spanning a time frame from June $8^{\text {th }} 2019$ up to March $15^{\text {th }} 2020$. In total the organization shared data from three estimators. For our analysis, only data from activities larger than 0.5 hours were considered: the very small user stories hold very large relative deviations and are of less interest to practitioners. Data from 1738 estimations remained.

For the first hypothesis, tasks were split into two categories: the user stories that took more than two hours to complete were considered 'large' tasks, the user stories that took less 'small'. Two hours was selected because the organisation's CEO considers all user stories that can be delivered within 2 hours to be small. For both the small and the large category, a boxplot was made indicating the plan accuracy of the estimations. Plan accuracy is in our case defined as:

Plan accuracy $:=\left(\frac{\text { estimation }}{\text { actual }}-1\right) * 100 \%$

In this we followed the convention of the forecast/actual ratio from a study on the estimation quality of business value (Eveleens, et al., 2012). The formula was altered slightly based on practitioners' feedback: the number 1 was subtracted so that a positive percentage means over-estimation and a negative percentage means under-estimation. This turned out to be easier to use and interpret in daily praxis (in the paper on business value, above 1 means over-estimation and below 1 under-estimation).

The boxplots give a more qualitative idea of the difference in estimation precisions and spread. To quantitatively compare both categories, a t-test was used. Independence and normality of data are required for a t-test. Data were assumed to be independent as they were on different user stories requiring new software. Because both categories have big sample sizes $(>500)$, their mean-values could be assumed to be continuous and we also assumed it to follow a normal distribution.

Also the second hypothesis was tested by splitting the data into two categories. The organisation creates software in both SQL as well as in Typescript, and user stories were categorised accordingly. As the estimators in the company do regularly program SQL they are considered as 'experienced' on the user stories delivered using SQL. Since they do not or hardly program Typescript, they are considered not to be experienced on those user stories. For hypothesis 2 the same method will be used as for hypothesis 1 (box plot and a t-test).

Hypothesis three was tested by fitting a linear regression model to the observed data. Two different models were made: one based on the monthly mean, the other on the monthly median. The significance of the fitted regressions were tested using t-tests, where the null hypothesis was zero slope (no regression) and the alternative hypothesis a positive slope (improvement).

\section{RESULTS}

We begin the section with some overall statistics on the data that was analysed.

The average time spend on a user story was 5.2 hours. $75 \%$ of all user stories were delivered within 6.2 hours. The outliers are user stories consuming $69.4,57.0$ and 48.8 hours, and the three largest estimations were 74.0, 40.0 and 24.0 hours.

Accuracy of estimations was calculated as mentioned above using the formula

$$
\text { Plan accuracy }:=\left(\frac{\text { estimation }}{\text { actual }}-1\right) * 100 \%
$$

The mean of the inaccuracy over the complete dataset is $-13.1 \%$, indicating an overall under-estimation. All estimations in the second quartile contain an underestimation of at least $-66.7 \%$. The estimations in the third quartile hold an overestimation of up to $5.3 \%$. The borders of the quartiles indicate that the bulk of estimations was too low. 


\subsection{Results for $\mathrm{H} 1$}

Comparing small and large user stories for testing H1 resulted in data shown in Figure 1. In the analysis, 644 of the stories were small, the remaining were big.

From the boxplot and the accompanying distribution data, it becomes visible that big tasks are more commonly under-estimated than small tasks. The mean estimation error for small tasks was $+42.36 \%$, for big tasks it was $-37.95 \%$. Therefore, in terms of sample means, there does not seem to be an absolute advantage for small tasks. The median for small tasks (6.67\%), however, lies much closer to zero than the median for big tasks (-52.38\%). This indicates that small tasks are under-estimated roughly as frequently as over-estimated. The spread in estimation errors is bigger for small tasks than for big ones, which can be explained by the fact that small errors in time are still large percentages of the smaller total time. A two-tailed Student's t-test performed on the data resulted in a small p-value, smaller than 0.001 hence there is a statistically significant difference between small and large tasks. We conclude that big tasks are under-estimated more frequently than small tasks, but we cannot accept $\mathrm{H} 1$, as we cannot conclude that small tasks are estimated better.

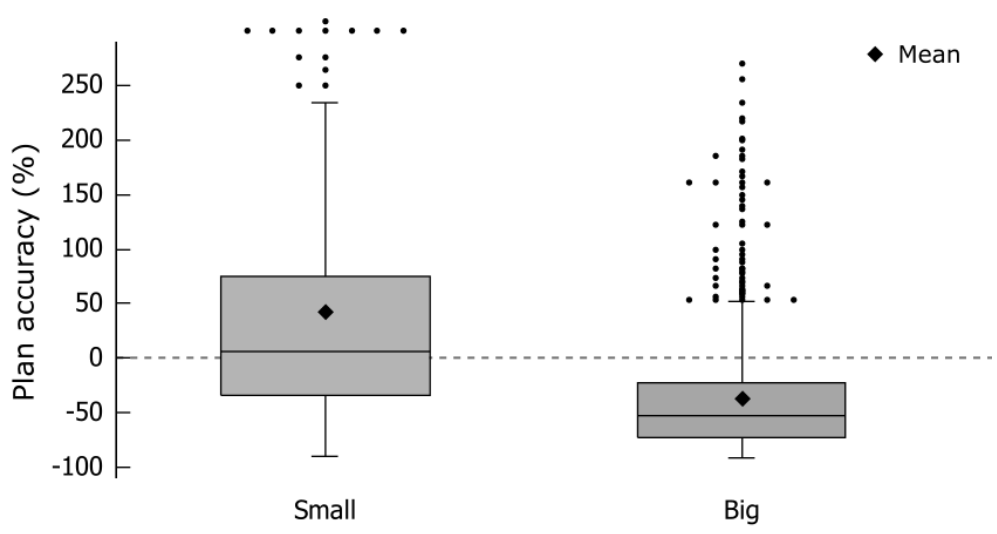

Figure 1. Comparing estimations for small and large user stories

\subsection{Results for $\mathrm{H} 2$}

In the boxplot in Figure 2 we present the difference between experienced and inexperienced estimations. Excluding outliers, we found 1454 User Stories where the estimators had experience in similar tasks, and 284 where they did not. The spread in errors is slightly smaller when the estimators could execute the task themselves. The mean error $(-16.65 \%)$ is much smaller than the mean error for when they could not deliver the user story $(5.69 \%)$. A two-tailed Student's t-test performed on the data resulted in a p-value smaller than 0.01 , so the difference is statistically significant. Interestingly, it seems that experienced estimations are on average worse. Most of the spread lies under zero, meaning under-estimations. This could be explained by the expert's blind spot. We cannot accept $\mathrm{H} 2$ that experienced estimations are better. It can be concluded, however, that user stories are more often under-estimated when the estimator could execute them him/herself. 


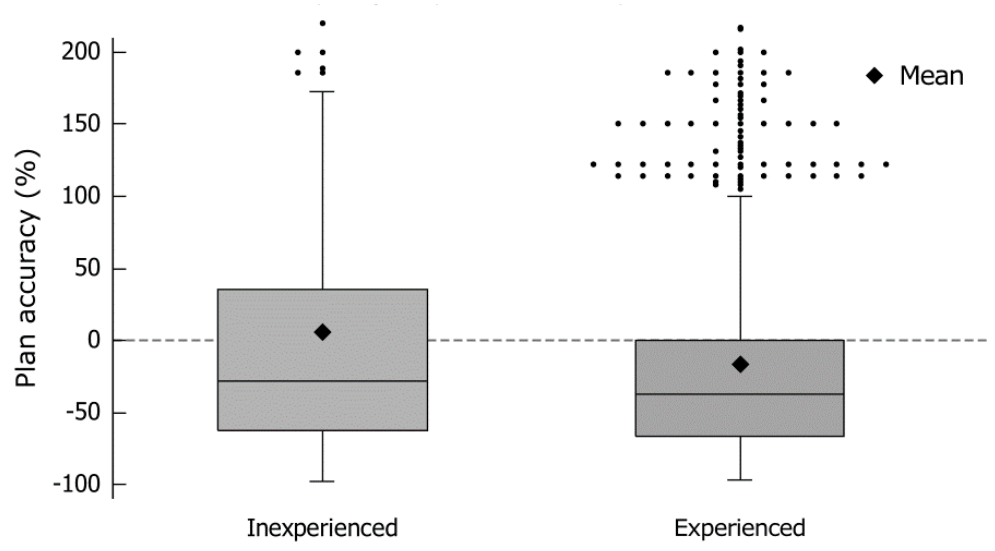

Figure 2. Comparing inexperienced and expert estimations

\subsection{Results for $\mathrm{H3}$}

The data used for H3 is visualised in Figure 3. The amount of datapoints per month are displayed in Table 1. By eye, estimations seem to improve: averages and medians get closer to zero. The spread in errors does not visibly improve.

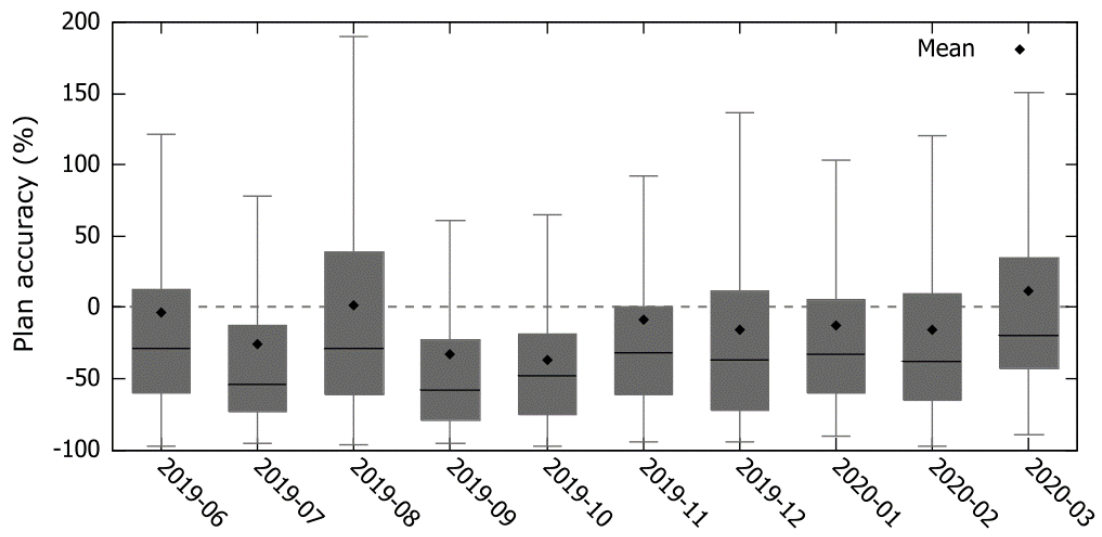

Figure 3. Development of accuracy over time

Table 1. Number of datapoints per month

\begin{tabular}{|ll|ll|}
\hline 2019/06: & 313 & $2019 / 11:$ & 161 \\
\hline $2019 / 07:$ & 229 & $2019 / 12:$ & 106 \\
\hline $2019 / 08:$ & 247 & $2020 / 01:$ & 148 \\
\hline $2019 / 09:$ & 128 & $2020 / 02:$ & 186 \\
\hline $2019 / 10:$ & 132 & $2020 / 03:$ & 88 \\
\hline
\end{tabular}

In order to test for improvement, the daily average plan accuracy was plotted and a linear regression model was fitted. Results are shown below in Figure 4. A slight improvement is visible: the difference between the fitted accuracy and 0 (no error) decreases over time. A t-test was performed to determine the significance of this fit. A p-value of 0.17 was found, meaning the improvement is not significant and also $\mathrm{H} 3$ must be rejected. 


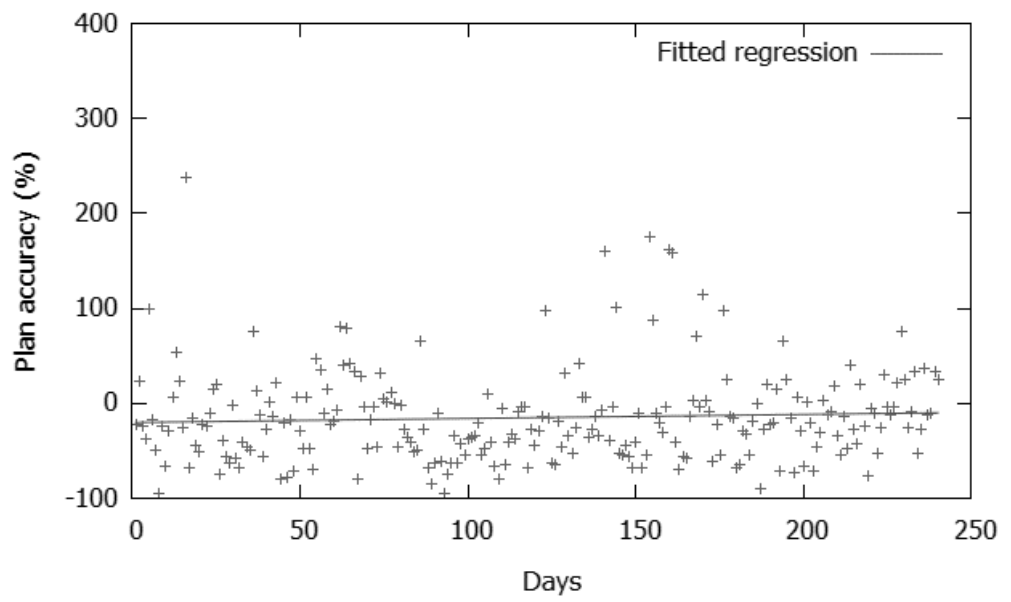

Figure 4. Mean plan accuracy per day

Table 2. Overview of hypotheses and results

\begin{tabular}{|c|c|c|c|}
\hline H1 & $\begin{array}{c}\text { Plan accuracy of time estimations for <2h user stories are } \\
\text { more accurate than those for larger user stories. }\end{array}$ & Rejected & Rejected \\
\hline H2 & $\begin{array}{c}\text { The plan accuracy of estimations is higher in case the } \\
\text { estimator can execute the task himself. }\end{array}$ & Rejected & \\
\hline H3 & $\begin{array}{c}\text { Plan Accuracy of estimations of an estimator improves over } \\
\text { time. }\end{array}$ & \\
\hline
\end{tabular}

\section{CONCLUSION}

The data studied in this paper does not shine a positive light on estimation accuracy: large inaccuracy was found across different types of user-stories. However, statistically significant differences were found between them.

It cannot be concluded that user stories $<2$ hours are estimated better than larger user stories. It did become clear, however, that large user stories are more commonly under-estimated, whereas estimations for smaller user stories are more 'balanced'.

It also could not be concluded that experienced estimations were better than inexperienced estimations. However, our analysis showed that experienced estimations were in general too low suggesting that the expert blind spot should not be underestimated in professional environments.

H3 was also rejected, meaning estimators do not improve their estimation accuracy on their own. This suggests more elaborate feedback should be given, a subject for further research.

We do think that the results acquired can be of value for practitioners: they give a more detailed insight into where weaknesses lie in making estimations. Furthermore, we still think it is possible to increase plan accuracy by providing better feedback, for which we make some suggestions in the next section.

To support practitioners, we present in (see for example Van der Pas and Furneaux).

Table 3 the combinations of the $\mathrm{H} 1$ and $\mathrm{H} 2$ parameters. This model builds four simple reference classes that can be used by practitioners to further improve predictions (see for example Van der Pas and Furneaux (2015)).

Table 3. Effect of different combinations of parameters

\begin{tabular}{|l|l|l|}
\hline & Estimated by non-expert & Estimated by expert \\
\hline $\begin{array}{l}\text { Smaller-than-two-hours user } \\
\text { story }\end{array}$ & Mean: $95.4 \%$ & Mean: $28.9 \%$ \\
\hline Larger-than-two-hours user story & Median: 46.4\%\% & Median: $0 \%$ \\
& Median: $-45.5 \%$ & Mean: $-42.3 \%$ \\
\hline
\end{tabular}


Additional instruments for improving the accuracy are described in the next section as suggestions for future research.

\section{LIMITATIONS AND SUGGESTIONS FOR FUTURE RESEARCH}

This research was focussed on small user stories (5.2 hours on average). The conclusions from this study cannot be projected on the estimation of large-scale IT projects consuming multiple years of effort and millions of Euros of investment.

There is a shortcoming of the used definition of plan accuracy in combination with the many small user stories. The problem with small tasks is that, with the current definition, small deviations result in big percentages. These percentages do not necessarily reflect how humans perceive: for example, in day-to-day tasks the difference between 5 and 6 minutes is considered to be unimportant, while the difference between 5 and 6 hours is seen as significant. Their percentage difference is the same, however. This non-intuitive behaviour should be kept in mind when studying the results.

When starting with this study, the estimators had limited experience in estimating user stories. The experience was cumulated fast with the start of this study in June 2019. The existing experience of the estimators should be taken into account when projecting the findings to other environments.

The current feedback the software company provides on estimations is simply a message showing the time estimated versus the time taken. Besides this case by case feedback, there was no statistical feedback. Feedback could be given by providing personalised, monthly estimation statistics like presented in the boxplot in

Figure 3, giving a better idea of structural errors. It could also be in the form of a boxplot presenting the actual time written. The latter gives insights to the average, mean as well as the spread of past user stories and could be used by the estimator as a guidance for future estimations. Also adding reference classes describing user stories realised to the plan accuracy feedback could improve the estimators learning curve. For the software organisation this could be reference classes on components they are programming such as 'workflow', 'reporting', 'investment management', 'scorecard', 'product catalogue' or 'agile performance management'.

On top of statistical feedback and reference classes, machine learning could be applied to push estimation quality. E.g. the techniques (van der Pas \& van der Pas, 2020) used to predict the likelihood of project cancellation could also be used to calculate an estimation as an output parameter. Input features for this estimation could be the initiator, the person who delivers the user story but also the already present data on the kind of software to be delivered. The machine learning prediction of the estimation could be used as an input parameter for the estimation made by the estimator.

Furthermore, future research could be done around the transparency of the estimation. The software company decided not to open the estimation to the person actually working on the user story. The reason for this is that the estimation could have a self-fulfilling effect on the person delivering the user story. It could make her relaxed in case she thinks that the work can easily be delivered in the estimated time or put pressure when the estimated time seems too little. This is contradictory to Goldratt who expects an open discussion to improve the estimation quality.

Finally, theories from social science can be used to influence the behaviour of estimators. Ajzen (Ajzen, 1991) developed the Theory of Planned Behaviour to predict behaviour of human beings. It was shown (van der Pas \& Walczuch, 2018) how, amongst others, intention and subjective norm can be used for this purpose. Asking estimators to confirm their intention on good estimations as well as adding automated plan reminders is likely to push the quality of their estimation (see e.g. Wicaksono, et al., 2019). Likewise, their intention for creating good estimations is likely to improve by increasing the subjective norm. The latter can be done by talking about and explaining the importance of estimations.

\section{ACKNOWLEDGEMENT}

The authors wish to thank Dr. Tobias Hartwich for his meaningful contribution towards designing the statistical methods to test the three hypotheses presented in this article. 


\section{REFERENCES}

Ajzen, I., 1991. The theory of planned behavior. Organizational behavior and human decision processes, 50(2), pp. $179-211$.

Ambrose, J., 2019. Smart energy meter rollout deadline pushed back to 2024. The Guardian, 16 September.

Bilal, K., Khan, W., Arshad, M. \& Jan, N., 2020. Software Cost Estimation: Algorithmic and Non-Algorithmic Approaches. International Journal of Data Science and Advanced Analytics, Issue 2.2, pp. 1-5.

Boehm, B. W., 1984. Software engineering economics. IEEE transactions on Software Engineering, Issue 1, pp. 4-21.

Eveleens, J. L., Van Der Pas, M. \& Verhoef, C., 2012. Quantifying forecast quality of IT business value. Science of Computer Programming, 77(3), pp. 314-354.

Farrel, S. \& Topham, G., 2019. Crossrail faces further delays and will cost more than £18bn. The Guardian, 8 November.

Goldratt, E. M., 2004. The goal : a process of ongoing improvement.. Great Barrington, MA: North River Press.

Hoffmann, D., Ahlemann, F. \& Reining, S., 2020. Reconciling alignment, efficiency, and agility in IT project portfolio management: Recommendations based on a revelatory case study. International Journal of Project Management, 38(2), pp. 124-136.

Jørgensen, M., 2004. A review of studies on expert estimation of software development effort. Journal of Systems and Software, 70(1-2), pp. 37-60.

Lauesen, S., 2020. IT Project Failures, Causes and Cures. IEEE Access, Volume 8, pp. 72059-72067.

Levin, A. \& Schlangenstein, M., 2020. New Software Flaw Could Further Delay Boeing's 737 Max. Bloomberg, 17 January.

Mahmood, Y., Kama, N. \& Azmi, A., 2020. A systematic review of studies on use case points and expert-based estimation of software development effort. Journal of Software: Evolution and Process, p. 2245.

The Standish Group, 2015. Chaos Report, s.1.: The Standish Group International, Inc.

van der Pas, M. \& Furneaux, B., 2015. Improving the predictability of IT investment business value. Münster, ECIS 2015 Completed Research Papers. Paper 190.

van der Pas, M. \& van der Pas, N., 2020. Improving Gate Decision Making Rationality with Machine Learning. Cham, Springer.

van der Pas, M. \& Walczuch, R., 2018. Behavior of Organizational Agents on Managing Information Technology. Cham, Springer, pp. 774-788.

Wicaksono, A., Hendley, R. \& Beale, R., 2019. Investigating the Impact of Adding Plan Reminders on Implementation Intentions to Support Behaviour Change. Interacting with Computers, 31(2), pp. 177-191.

Wiggins, G. \& McTighe, J., 2005. Understanding by Design. Expended 2nd edition ed. s.1.:Association for Supervision and Curriculum Development. 